Hydroécol. Appl. (2001) Tome 13 Vol. 2, pp.149-173

\title{
Etude des impacts cumulés sur l'Isère en aval de la retenue d'Aigueblanche (Moutiers - France)
}

\section{Cumulative impacts assessement on the Isere River downstream the Aigueblanche reservoir (Moutiers - France)}

\author{
A. Poirel (EDF/DTG) \\ 21, av de l'Europe - BP41 - 38040 Grenoble Cédex \\ E-Mail : alain.poirel@edf.fr
}

Résumé. - L'étude environnementale des chasses hydrauliques sur la retenue d'Aigueblanche (Isère) montre la difficulté d'interpréter les données physico-chimiques et biologiques sur une rivière fortement anthropisée. Les différents impacts sont analysés individuellement, puis en fonction de leurs synergies et antagonismes. La disparité des informations disponibles selon la nature des impacts rend difficile cette analyse pour un acteur particulier. Seule une stratégie concertée entre tous les acteurs permettrait d'expliquer les variations biologiques observées sous réserve de les situer dans le cadre de la forte variabilité naturelle de ces écosystèmes montagnards.

Mots-clés. - Retenue, Chasse, Impacts environnementaux, Cumul, Isère.

\begin{abstract}
The environmental study of hydraulic flushing on the Aigueblanche reservoir (Isère-France) shows the difficulty to interpret physicochemical and biological data on a highly anthropized river. The various potential impacts are analysed individually, then according to their interactions. Available data are scattered within several stakeholders, making difficult for each of them taken individually to raise conclusions. A global framework involving a shared analysis of data would the understanding of biological impacts possible, by taking into account the high natural variability of such mountain ecosystem.
\end{abstract}

Key words. - Reservoir, Flushing, Environmental impacts, Cumulative effects, Isère river. 


\section{INTRODUCTION}

Dans le cadre des chasses effectuées sur la retenue d'Aigueblanche, de nombreuses mesures physico-chimiques et biologiques ont été réalisées au cours de 11 opérations réparties sur la période $1983-2001$. Une synthèse de ces informations [Bovio \& Poirel, 1998] a montré la quasi-impossibilité d'interpréter les relations entre mesures physico-chimiques lors des chasses d'une part et mesures biologiques d'autre part. La présente communication a pour objectif de montrer la difficulté pour un acteur particulier à appréhender le cumul de tous les impacts lors de l'analyse des données biologiques. Dans une rivière aussi anthropisée que I'Isère entre Moutiers et Albertville, l'étude simultanée de tous les impacts, quelle que soit leur nature, apparaît comme une phase incontournable pour l'interprétation des données biologiques.

\section{PRÉSENTATION DU SITE}

La retenue d'Aigueblanche est située au confluent de l'Isère et du Doron de Bozel. Sa cote à « retenue normale " est de 470,5 m NGF mais son bassin versant est nettement plus élevé avec une altitude moyenne de $2150 \mathrm{~m}$ pour $1582 \mathrm{~km}^{2}$. Les pentes de l'Isère et du Doron en amont sont respectivement de $1,1 \%$ et de $2,5 \%$. Le module de l'Isère à Aigueblanche est de $55 \mathrm{~m}^{3} / \mathrm{s}$ [Tourasse, 1989].
Le barrage est placé sur un resserrement de la vallée (les Echelles d'Hannibale) qui crée une pente de plus de $3 \%$ dans les gorges d'Aigueblanche. En aval du barrage, la pente s'adoucit très nettement, tout d'abord à $0,6 \%$ puis à $0,5 \%$ en aval de $\mathrm{Ce}$ vins [Grandes Forces Hydrauliques, 1911].

A Aigueblanche, les eaux de l'Isère et du Doron sont dérivées vers la centrale hydroélectrique de Randens sur l'Arc dans la limite de $123 \mathrm{~m}^{3} / \mathrm{s}$. La retenue d'Aigueblanche sert également de bassin inférieur pour l'aménagement de Transfert d'Energie par Pompage de la Coche (débit maximum de $40,5 \mathrm{~m}^{3} / \mathrm{s}$ ). La figure 1 synthétise les points de mesures et la nature des mesures environnementales disponibles sur le tronçon.

\section{3 ÉVOLUTION DU CONTEXTE ENVIRONNEMENTAL}

\section{1 Évolution du contexte morphodynamique}

Le cours de l'Isère passe d'un système torrentiel à très haute énergie en amont d'Aigueblanche à un système moins pentu puis à une vallée plus ouverte en aval de Cevins. En réponse, la morphologie initiale de la rivière s'adapte, passant d'un lit en gorges étroites à un lit anastomosé.

Historiquement, les premiers impacts remontent au $14^{\text {ème }}$ siècle avec l'établissement des digues destinées à protéger routes et habitations des 


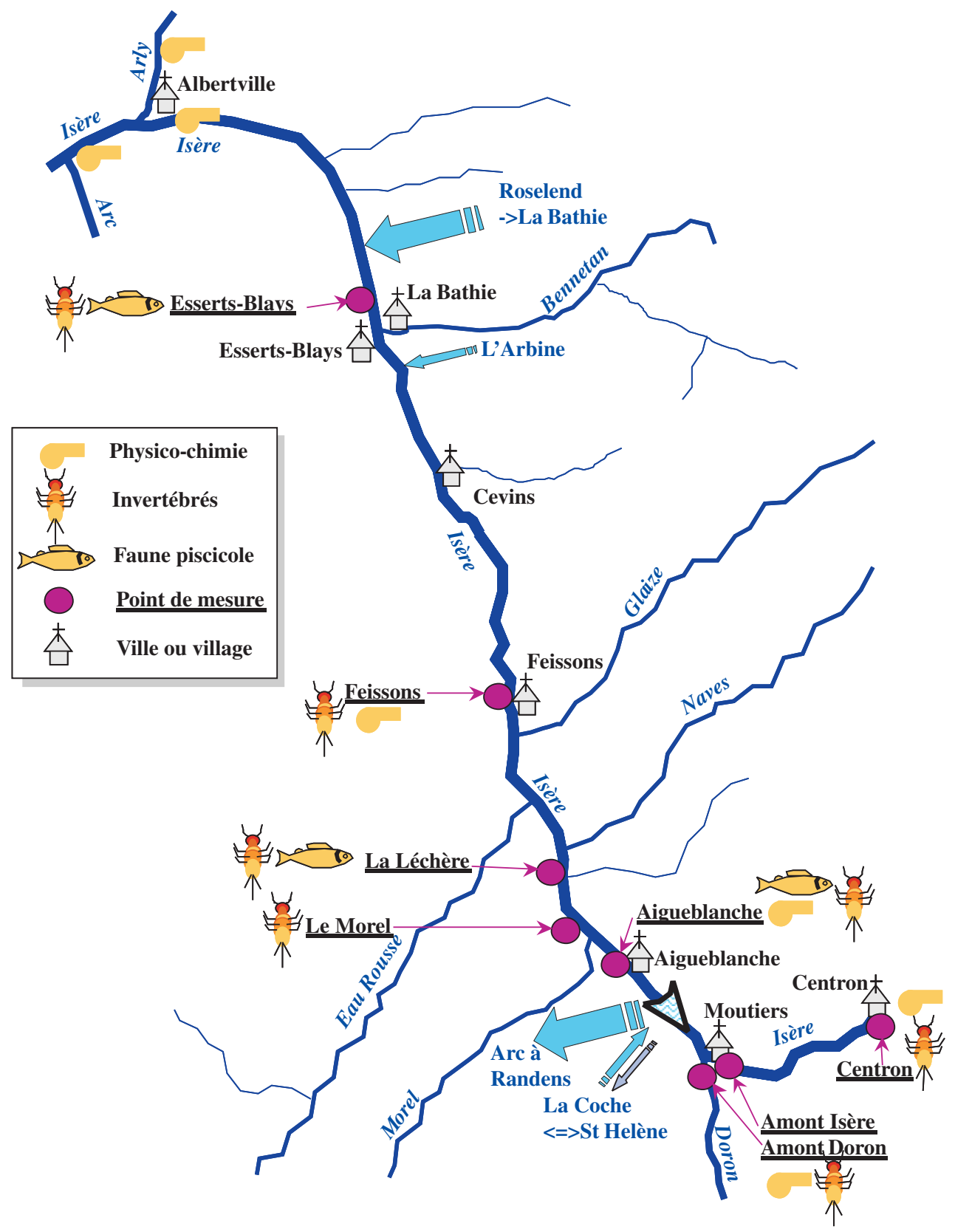

Fig. 1. - Vue générale du site d'étude et localisation des points de mesure.

Fig. 1. - Global overview and location of sampling stations. 


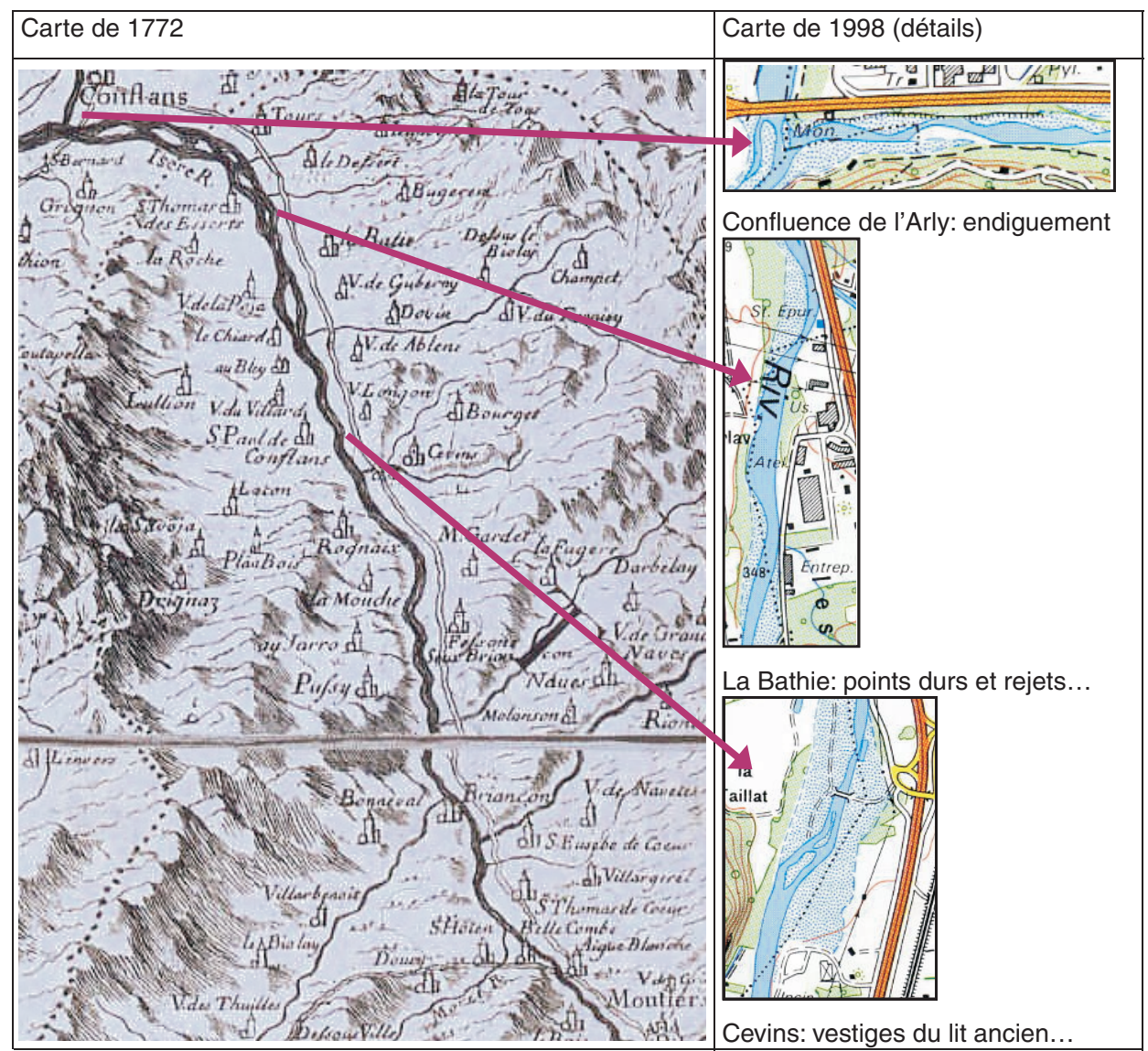

Fig. 2. - Evolution du contexte morphologique entre 1772 [Anville, 1697-1782] et 1998 [Carte IGN 3532 OT, 1998].

Fig. 2. - Morphological evolution of the river between 1772 [Anville, 1697-1782] and 1998 [Carte IGN 3532 OT, 1998].

crues de l'Isère. Ces digues se sont ensuite multipliées soit pour protéger des sites industriels qui avaient besoin d'eau, soit pour permettre le passage de nouveaux axes de communication. Cette tendance est toujours marquée aujourd'hui avec l'ajout régulier de nouveaux «points durs » limitant l'extension latérale de la rivière (Fig. 2).

Les conséquences majeures de cet endiguement sont la réduction de la largeur du lit, l'augmentation corollaire des hauteurs et des vitesses, particulièrement en crue, et une uniformisation des écoulements. À la station 
Relations en moyenne sur la section Débit / Vitesse et Débit / Hauteur à Cevins
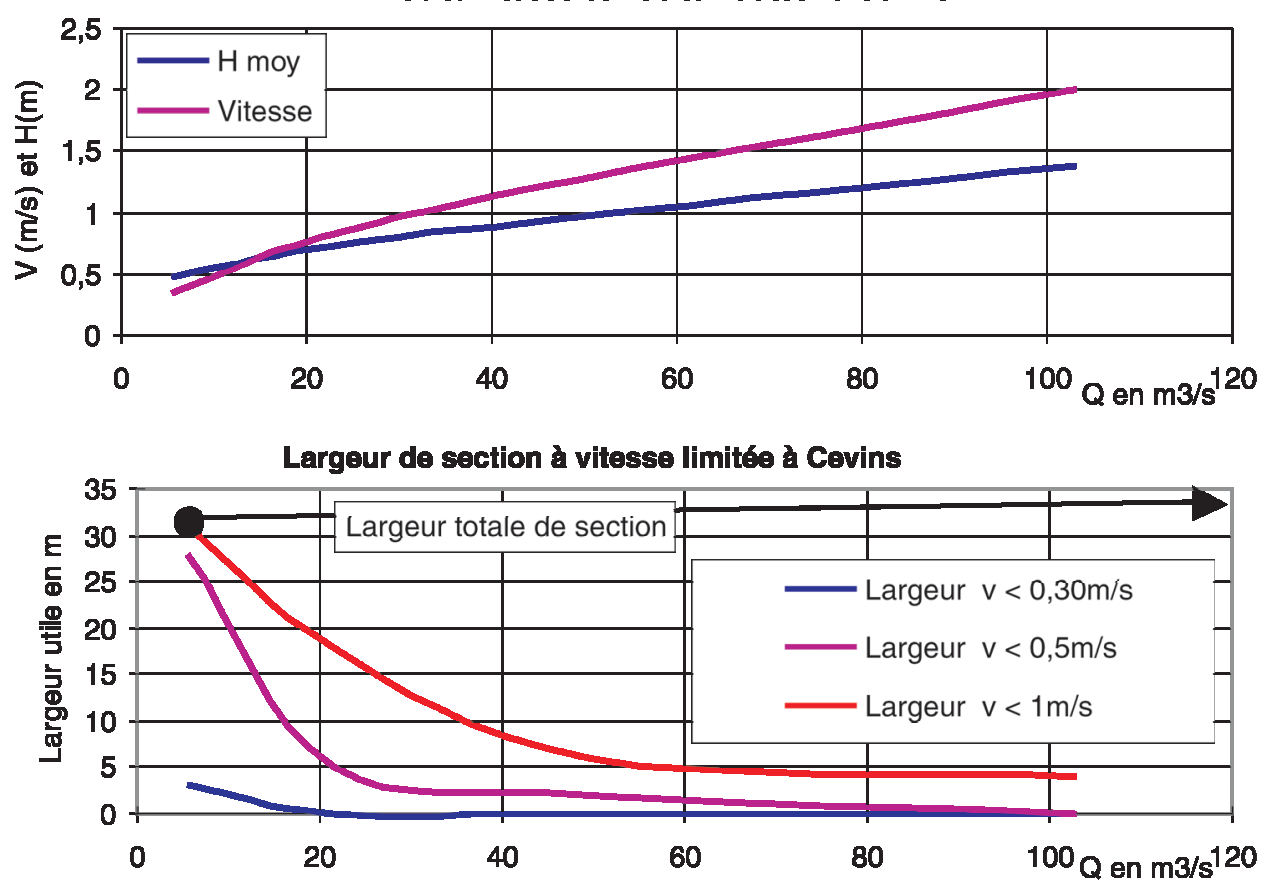

Fig. 3. - Relations entre le Débit, la Vitesse, la Hauteur et différentes Largeurs « utiles » (largeur où la vitesse est inférieure à une valeur donnée) à la station limnimétrique de Cevins.

Fig. 3. - Relations between flow, speed, water level and various « useful » widths (width where speed is lower than a given value) at the gauging station of Cevins.

limnimétrique de Cevins, par exemple, lorsque le débit passe de 5 à $50 \mathrm{~m}^{3}$ par seconde, la largeur de la rivière ne passe que de 31 à 34 mètres. Parallèlement, la vitesse moyenne passe de 0,4 à $1,3 \mathrm{~m} / \mathrm{s}$ et la largeur de rivière où la vitesse est inférieure à $0,5 \mathrm{~m} / \mathrm{s}$ n'est plus que de $2 \mathrm{~m}$ (Fig. 3).

De plus, nombre d'affluents ne peuvent plus constituer une zone de refuge ou de reproduction pour les poissons (Fig. 4), soit parce qu'inac- cessibles suite à l'incision du lit de I'Isère (confluences perchées), soit à cause de vitesses trop élevées (pentes naturellement fortes et nombreuses rectifications torrentielles). Les impacts de cette chenalisation sur la faune piscicole sont bien décrits dans [Wasson et al., 1995]. Des effets irréversibles sur la biomasse, atteignant parfois $80 \%$, sont signalés, affectant essentiellement les espèces halieutiques. 
Vue cartographique de la confluence du Morel avec l'Isère en 1908 : le cône de déjection du torrent en forme de delta repousse l'Isère, la confluence est formée de différents bras, une île est figurée sur l'Isère qui semble élargie.

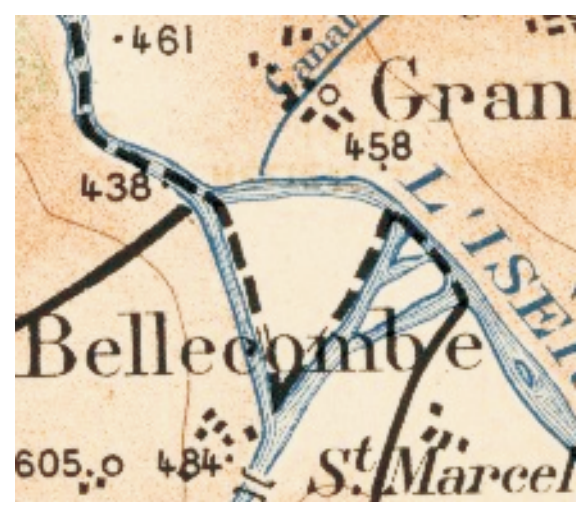

Vue cartographique de la confluence du Morel avec l'Isère en 1998 : le Morel est complètement endigué en un seul bras, le lit est maintenu par des seuils anti-érosion, le delta, seule zone relativement plate a été aménagé en base de loisirs...

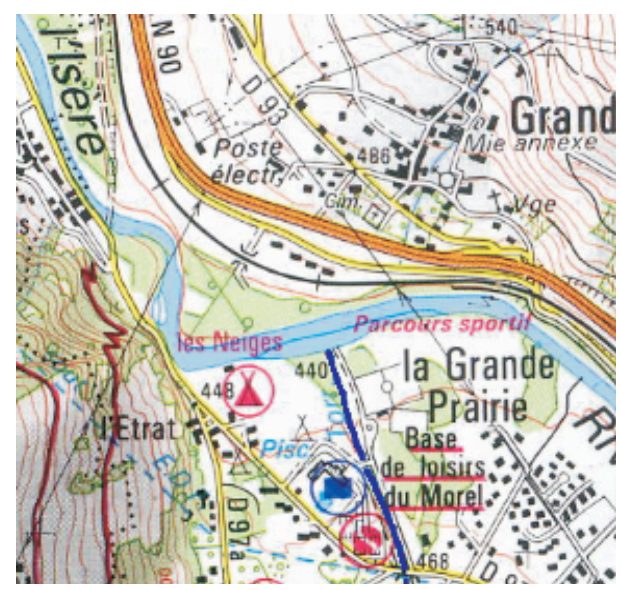

Fig. 4. - Evolution de la confluence du Morel entre 1908 [Grandes Forces Hydrauliques, 1908] et 1998 [IGN, 1998].

Fig. 4. - Evolution of the confluence of Morel river and Isere River between 1908 [Grandes Forces Hydrauliques, 1908] and 1998 [IGN, 1998].

\subsection{Evolution du contexte climatologique}

La vallée de l'Isère a été soumise ces dernières années à des évolutions notables en matière de température de l'air. Les moyennes interannuelles à Bourg-Saint-Maurice, de 8 à $9^{\circ} \mathrm{C}$ sur la période 1977-1987, sont passées de 9,5 à $10,5^{\circ} \mathrm{C}$ sur la période récente 1989-2000 (Fig. 5).

En outre, une analyse plus fine indique que la saison printanière est de loin la plus impactée avec presque + $3^{\circ} \mathrm{C}$ d'écart entre les deux périodes. C'est naturellement une saison très sensible pour les écosystèmes alpins avec l'émergence des alevins de truite et le développement maximal de la faune (Fig. 6).

\subsection{Régime hydrologique}

Le régime hydrologique de l'Isère est fortement modifié par l'exploitation hydroélectrique selon 3 composantes : Ia modification annuelle des écoulements avec un report des eaux de fonte vers l'hiver suivant, la mise en débit réservé du tronçon à l'aval d'Aigueblanche jusqu'à la confluence avec l'Arc à l'aval d'Alberville, le régime d'éclusées sensible en amont 


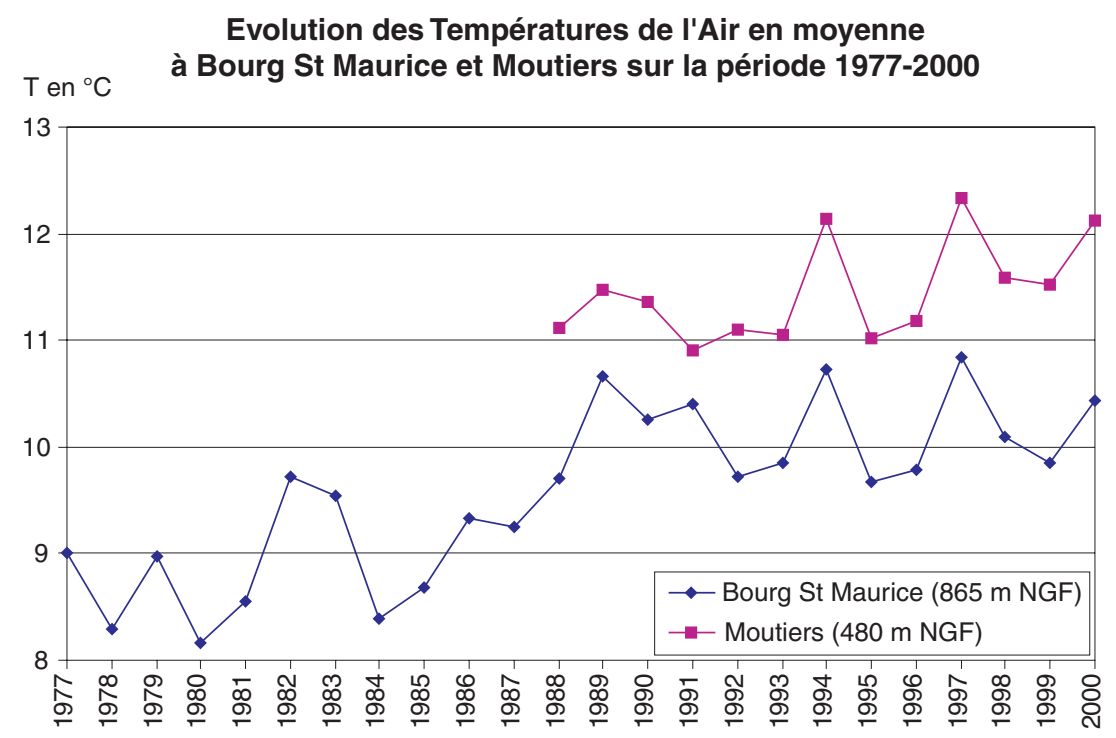

Fig. 5. - Evolution des températures de l'air en moyenne annuelle aux stations de Bourg-SaintMaurice et Moutiers de 1977 à 2000.

Fig. 5. - Evolution of yearly mean air temperature in Bourg-Saint-Maurice and Moutiers from 1977 to 2000 .

\section{Evolution des moyennes saisonnières comparées à la moyenne saisonnière interanuelle}

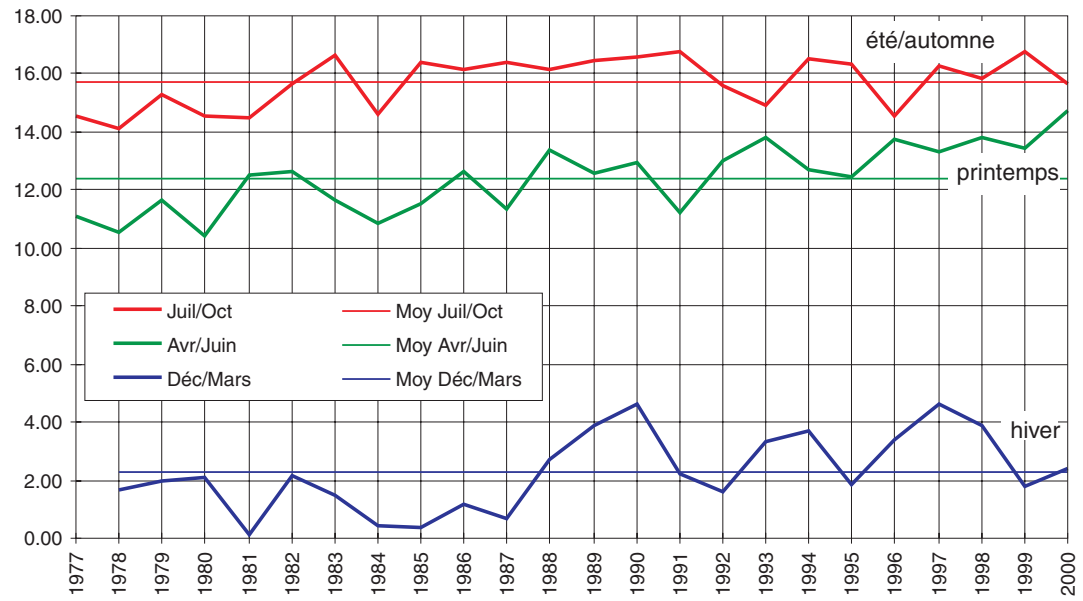

Fig. 6. - Evolution des températures moyennes par saison à la station de Bourg-Saint-Maurice de 1977 à 2000.

Fig. 6. - Evolution of mean air temperatures by season in Bourg-Saint-Maurice from 1977 to 2000. 
d'Aigueblanche ainsi qu'en aval de l'usine de la Bathie débouchant aux deux tiers du tronçon court-circuité.

A l'aval du tronçon, à Albertville, l'hydrologie est donc très complexe avec les influences précédentes auxquelles il convient d'ajouter l'hydrologie naturelle des affluents et les déversements à Aigueblanche. Ces déversements n'interviennent que sur des crues exceptionnelles lorsque la prise d'eau de Randens est saturée ou lors des chasses, différenciant de manière très nette les années où ils ont lieu (Fig. 7).

II est probable que le réchauffement des températures de l'air a des impacts sur la période de fonte [Paquet \& Garçon, 2001], même si les ré- percussions sur le régime des débits ne sont pas faciles à mettre en évidence en raison de l'aménagement hydroélectrique de la vallée. Les répercussions de cette évolution sur les écosystèmes, soit au travers des températures de l'eau, soit par le décalage plus tôt dans l'année des crues de fusion printanières, sont encore très mal connues. On sait cependant que les cycles vitaux de certains organismes sont liés à la fois à la photopériode et à la température de l'eau [Poirel et al., 2001].

\subsection{Régime du transport solide en suspension et par charriage}

Lors de l'étude de retour d'expérience sur les chasses d'Aigue-

\section{Isère à Albertville}

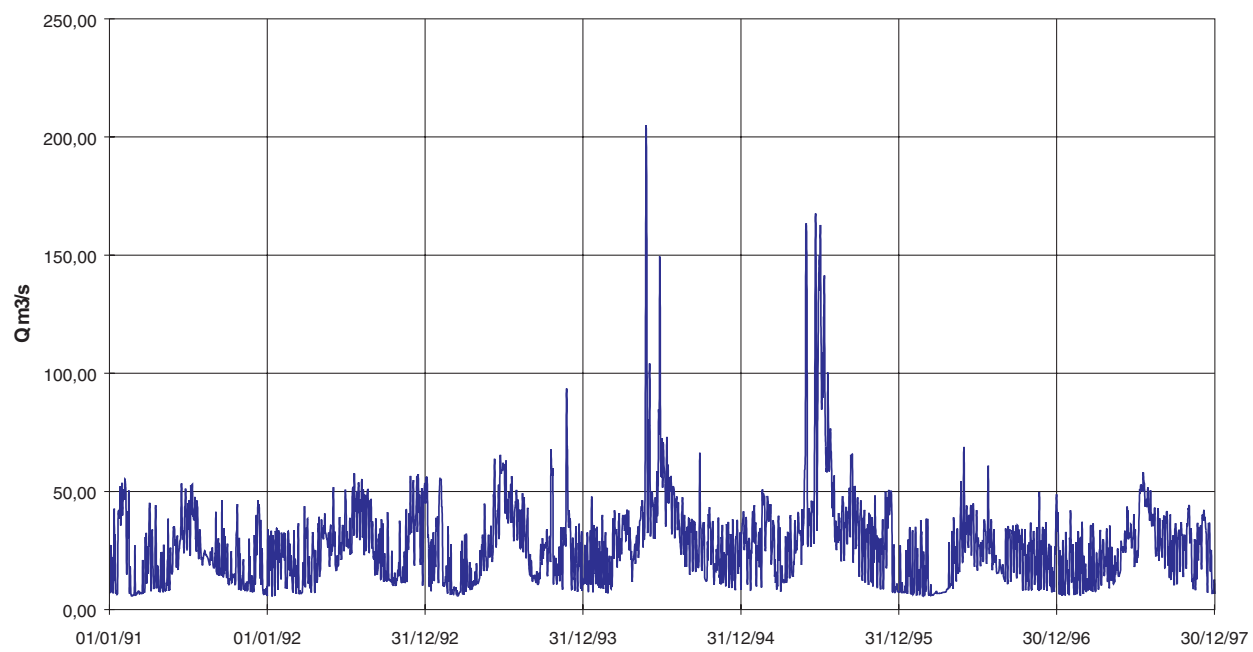

Fig. 7. - Chronique des débits moyens journaliers en aval de la zone d'étude (Station de Cevins + Turbinés de l'usine de la Bathie).

Fig. 7. - Daily mean discharge downstream the studied area (gauging station of Cevins + turbinate flow from La Bathie hydropower plant). 
blanche [Bovio \& Poirel, 1998], les apports en suspension via l'lsère et le Doron de Bozel ont été reconstitués à partir des relations MES/Débit journalier, calées sur les observations faites en amont de la retenue lors des chasses. Ces valeurs correspondent aux apports naturels en suspension des bassins versants amont, à l'exception de celui de la haute Isère en amont de Tignes. Cette reconstitution a cependant ses limites car certains épisodes faisant intervenir des laves torrentielles sont très mal représentés par cette approche. Aucune mesure de MES n'a été réalisée lors de tels événements, comme celui de Juillet 1996 sur l'Arbonne [Koulinski, 1999] très dévastateur au niveau de BourgSaint-Maurice, et évalué à 300000 tonnes (suspension et charriage).
Sur la période 1969-1997, le transport solide en suspension en entrée d'Aigueblanche s'établit à 250000 tonnes annuelles. Cette valeur moyenne cache cependant une très forte variabilité avec notamment une année estimée à plus de 900000 tonnes et quelques années à moins de 60000 tonnes. Ce régime solide important implique naturellement l'envasement de toutes les retenues positionnées sur le cours de I'Isère. La retenue d'Aigueblanche n'échappe pas à la règle même si sa capacité totale $\left(0,7 \mathrm{hm}^{3}\right)$ ne représente, en moyenne, qu'une à deux années de transport solide d'où un équilibre sédimentaire rapidement atteint (Fig. 8).

Les valeurs naturellement observées lors des crues en l'absence de

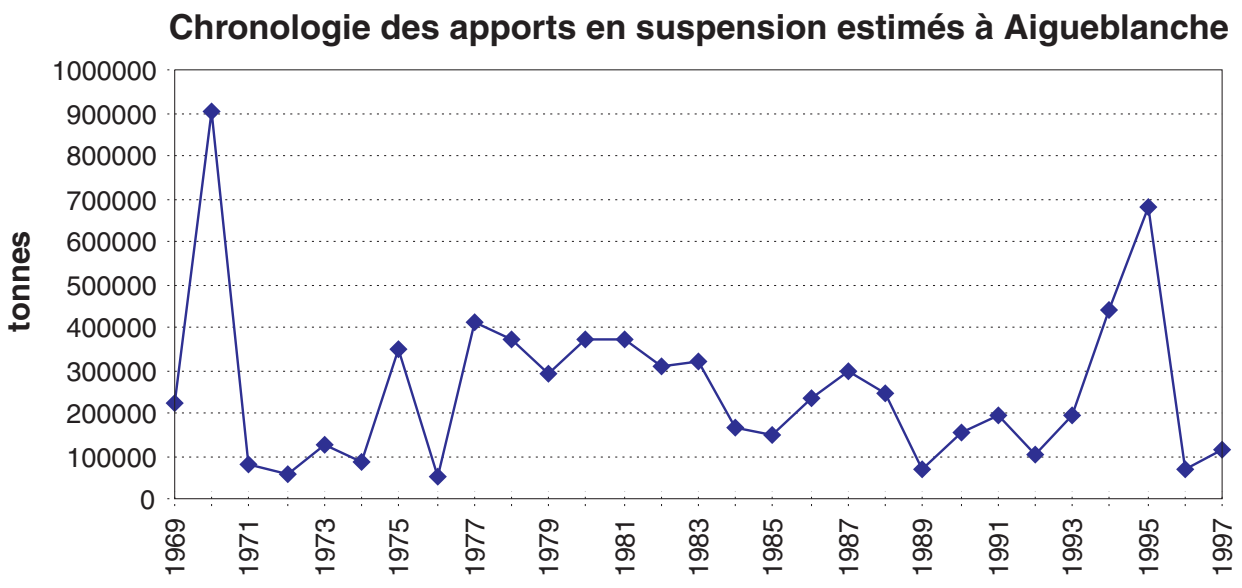

Fig. 8. - Chronique des apports solides en suspension reconstitués en entrée de la retenue d'Aigueblanche.

Fig. 8. - Modelized time series of suspended sediment discharge upstream the Aigueblanche reservoir. 
chasses sont rares. Cependant, lors de la crue du 12 mai 1999, on a mesuré $8,7 \mathrm{~g} / \mathrm{l}$ sur l'Isère amont, $3,8 \mathrm{~g} / \mathrm{l}$ sur le Doron, $7,7 \mathrm{~g} / \mathrm{l}$ en aval immédiat de la retenue et $7,1 \mathrm{~g} / \mathrm{l}$ à Albertville pour un débit moyen journalier de $208 \mathrm{~m}^{3} / \mathrm{s}$ en entrée d'Aigueblanche et $135 \mathrm{~m}^{3} / \mathrm{s}$ déversés au barrage. Encore s'agit-til d'une valeur isolée qui ne permet pas d'estimer les maxima lors de la crue.

Sur les affluents par contre, les valeurs semblent fréquemment dépasser $10 \mathrm{~g} / \mathrm{l}$ voire même $20 \mathrm{~g} / \mathrm{l}$ comme sur le Nant Brun et l'Eau Rousse [Poirel \& Bessy, 2000].

Les conséquences toxicologiques directes de ces concentrations en MES sont peu probables compte tenu de la durée des pics, mais à plus long terme, les dépôts successifs de matériaux fins peuvent avoir des conséquences par colmatage des habitats. L'évolution de ce colmatage est difficile à estimer car deux phénomènes se compensent : la mise en débit réservé du tronçon et l'arrêt rapide des débits sur les déversements au barrage (reprise de la dérivation vers Randens) contribuent à une moindre dilution des apports solides des affluents et un moindre entraînement des MES vers l'aval, alors que, a contrario, l'endiguement contribue au transit rapide des MES vers l'aval par l'augmentation de la vitesse et l'uniformisation du chenal (peu de zones de dépôt).

De plus, le régime du charriage est également fortement perturbé dans ce secteur. Des extractions en lit mineur ont été pratiquées soit pour des granulats, soit pour maintenir les lignes d'eau sur certains secteurs sensibles. Les résultats d'extraction en amont d'Aigueblanche donnent un régime moyen d'environ $30000 \mathrm{~m}^{3} / \mathrm{an}$ [Bovio \& Poirel, 1998]. En aval, les observations réalisées lors des prélèvements pour les IBGN montrent que les granulométries sont fortement variables dans le temps, probablement en liaison avec certains événements amont, telle la lave torrentielle de l'Arbonne, qui apportent des quantités importantes de matériaux en des temps très brefs.

\subsection{Régime et évolution de la physico-chimie des eaux}

Le régime annuel physico-chimique de l'Isère dans le tronçon court-circuité est établi à partir des données (1983, 89, 92, 95, 97 à 99) du Réseau National des Données sur l'Eau (RNDE). II se comporte à l'opposé du régime hydrologique. II traduit les rejets estivaux et surtout hivernaux liés au tourisme. Ces rejets hivernaux sont concomitants à l'étiage, même si l'exploitation hydroélectrique tend globalement à favoriser la dilution durant cette saison, surtout en aval de la restitution de la Bathie (Fig. 9).

Parmi les ions majeurs mesurés, les deux points fondamentaux suivants sont à noter :

- les sulfates constituent l'essentiel des cations avec des concentrations supérieures à $300 \mathrm{mg} / \mathrm{l}$; ils 


\section{Régime de quelques éléments chimiques majeurs sur I'Isère à Feissons

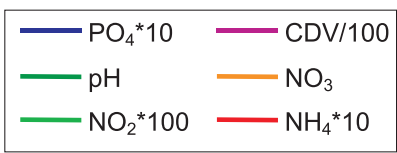

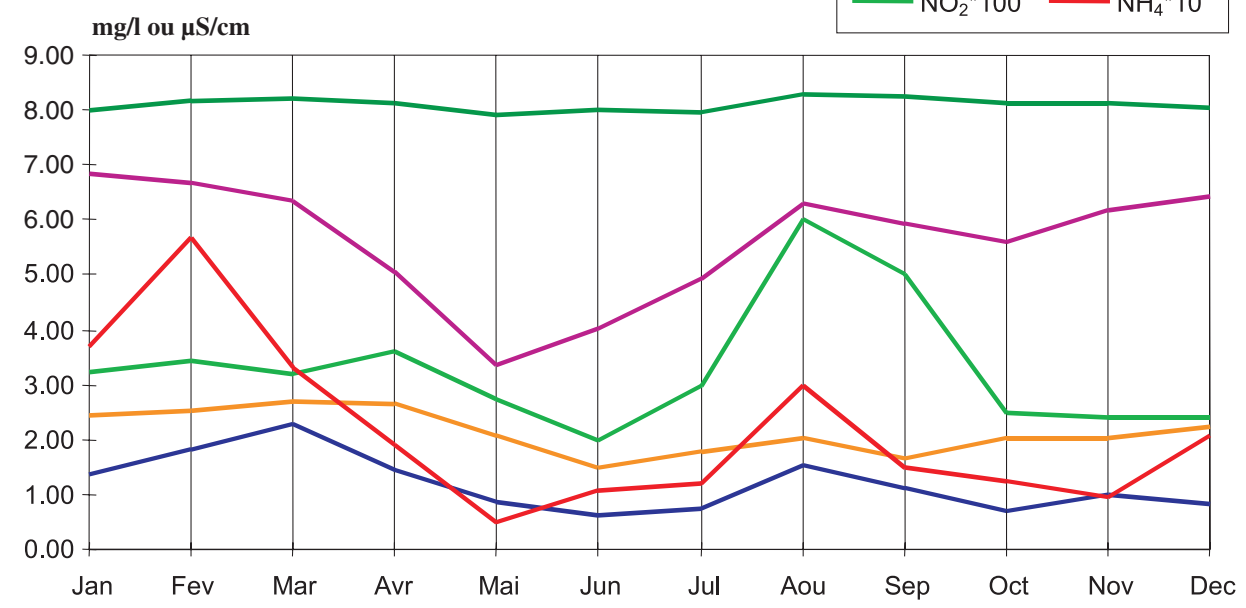

Fig. 9 - Régime en moyenne mensuelle de quelques éléments chimiques sur l'lsère (Feissons). ( $\mathrm{Cdv}=$ Conductivité, $\mathrm{NO}_{2}=$ nitrites, $\mathrm{NO}_{3}=$ nitrates, $\mathrm{NH}_{4}=$ ammonium, $\mathrm{PO}_{4}=$ phosphates $)$.

Fig. 9 - Monthly mean of some physicochemical parameters in the Isere river (Feissons).

sont liés au fond géochimique régional,

- l'azote ammoniacal présente des valeurs élevées avoisinant le $\mathrm{mg} / \mathrm{l}$ durant les mois de Janvier et Février 1997, 98 et 99 et un pic secondaire en été ; compte tenu du $\mathrm{pH}$ assez élevé $(8,2$ sur la mesure à $1,15 \mathrm{mg} / \mathrm{l}$ ) la formation d'ammoniac dissous n'est peut-être pas sans effet durant cette période hivernale où les œufs de truites sont dans les frayères. Le pas de temps des mesures disponibles (mensuel) ne permet pas d'estimer les valeurs extrêmes atteintes par ce paramètre mais la figure 10 laisse supposer que la situation est plutôt en train de se dégrader.
Les rejets industriels semblent surtout se traduire par une contamination métallique avec plusieurs paramètres en classe rouge ou orange du SEQ Eau (As, Hg, Cr, Pb). Cela ce traduit, par des indices SEQ-Eau dans les bryophytes de 6 à 60 . Si la situation est dégradée pour ces paramètres, elle est néanmoins en amélioration sensible depuis 1995 (fermetures de sites industriels, effort d'épuration). En amont de la retenue, à Centron, l'indice SEQ Eau - Métaux dans les bryophytes s'améliore notablement passant de la gamme 10-12 en 1988 à 50-60 en 1998-1999. En aval, la contamination globale décroît plus nettement mais un seul paramètre $(\mathrm{Cr})$ suffit à masquer ces gains 
L'Isère aval à Feissons / Isère

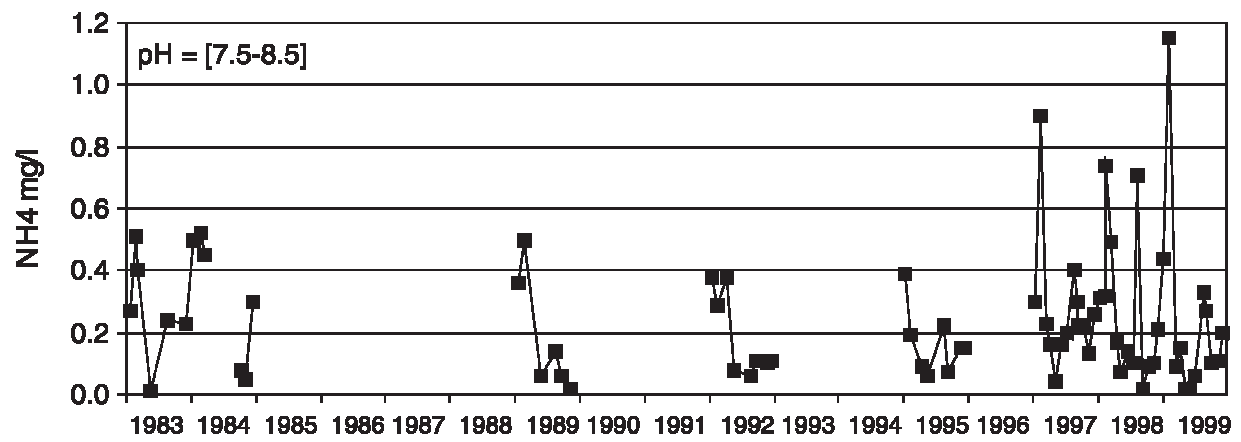

Fig. 10 - Evolution chronologique de l'ammonium sur l'Isère de 1983 à 1999 (Feissons).

Fig. 10 - Time series of ammonium in the Isere river (Feissons).

en février 1998 avec un indice SEQ à 19 alors qu'il est à 50 en 1999 (Fig. 11a et 11b).

Les rares mesures de métaux dans les sédiments indiquent des valeurs au dessus du PEL (Probable Effect Level) [Smith et al., 1996] et du PEC (Probable Effect Concentration) [Min. Equipement, 2001] pour l'arsenic, au dessus du PEL pour le nickel, au-dessus du TEL (Threshold Effect Level) pour le Cadmium et le Chrome.

\section{CONTEXTE PARTICULIER LIÉ AUX CHASSES}

Le transport solide à Aigueblanche est géré par des chasses hydrauliques (abaissement complet du plan d'eau lors d'une crue). Cette méthode permet de rétablir le transit solide durant un événement hydrologique majeur avec deux effets: l'évacuation d'une partie des sédiments déposés dans la retenue depuis la chasse précédente et le non-dépôt des sédiments apportés par la crue. Elle est efficace puisqu'elle permet de revenir à un volume de retenue voisin de $0,6 \mathrm{hm}^{3}$ pour une capacité initiale de $0,68 \mathrm{hm}^{3}$.

Dans l'état d'envasement maximum, après plusieurs années sans chasse possible à cause de l'hydraulicité, la retenue a une capacité de $0,4 \mathrm{hm}^{3}$. Elle stocke environ une année de transport solide moyen (300 000 tonnes). Au-delà, les apports solides entrants sont évacués soit par les débits turbinés dans l'Arc soit lors des déversements vers l'aval en crue. Cependant, l'exploitation du barrage est beaucoup plus délicate dans cette configuration d'envasement: cela peut se traduire par l'augmentation des risques de déversements impromptus sur des colmatages d'organes de prise. 
L'Isère amont à Centron
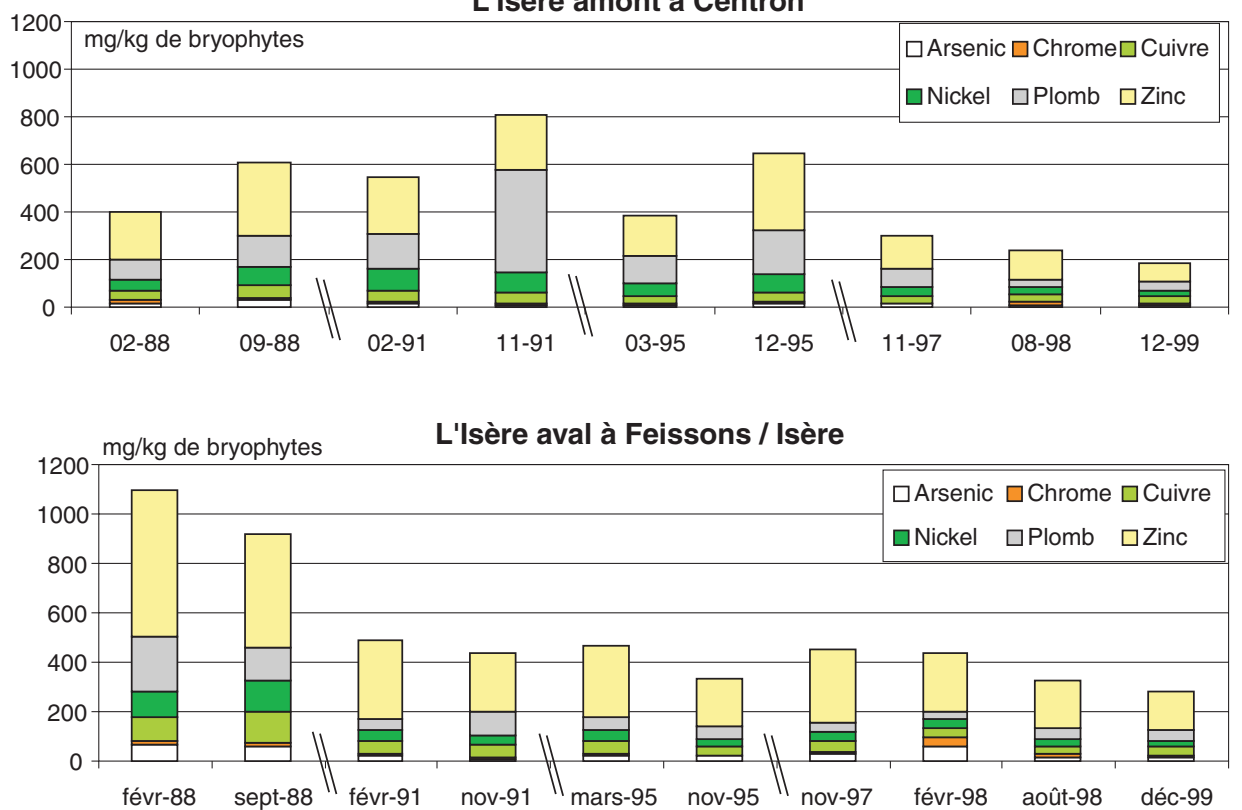

L'Isère amont à Centron

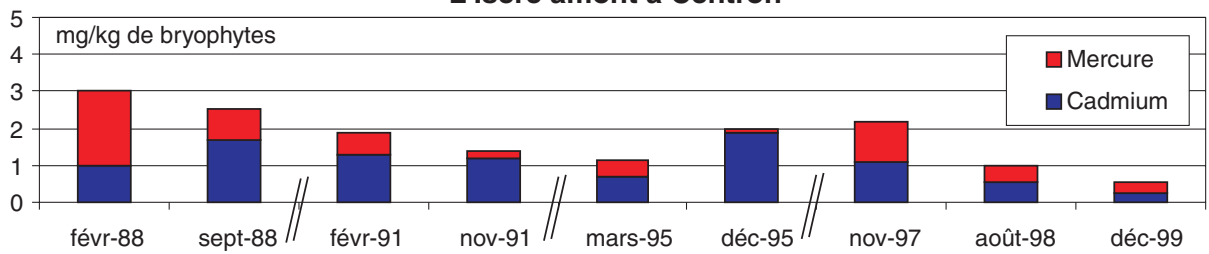

L'Isère Aval à Feissons

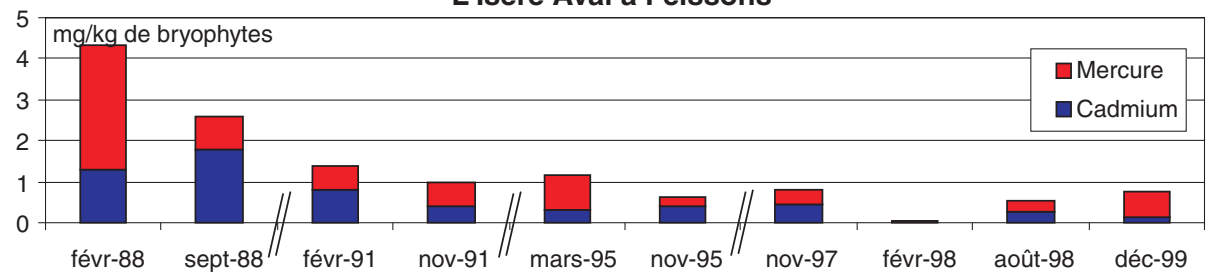

Fig. 11a. - Evolution chronologique des concentrations des principaux métaux lourds dans les bryophytes de l'Isère (As, $\mathrm{Cr}, \mathrm{Cu}, \mathrm{Ni}, \mathrm{Pb}, \mathrm{Zn})$ - Fig. 11b. - (Hg, Cd).

Fig. 11a. - Chronological evolution of heavy metals in bryophyte in the Isere river ( $\mathrm{As}, \mathrm{Cr}, \mathrm{Cu}, \mathrm{Ni}$, $\mathrm{Pb}, \mathrm{Zn})$ - Fig. 11b. - (Hg, Cd). 
Les chasses, contrairement aux autres événements pouvant avoir un impact marquant (crues, pollutions chroniques ou restreintes aux périodes touristiques, travaux d'endiguement), font l'objet de suivis spécifiques qui allient des mesures physico-chimiques à pas de temps fin, des mesures biologiques (IBGN et pêches avant et après opération). Les conditions initiales de débits, le gradient hydraulique maximal en début et en fin d'opération, le placement de la chasse par rapport à la crue ont été modifiés au cours du temps pour respecter les règles suivantes qui sont parfois contradictoires :

- choix d'une crue suffisamment importante pour permettre la dilution et l'entraînement des sédiments dans un grand volume d'eau (c'est donc plus le volume de la crue qui importe que sa pointe),

- choix d'une crue suffisamment fréquente pour éviter l'accumulation de sédiments sur plusieurs années,

- pas d'augmentation de la pointe de crue en aval,

- minimisation des gradients hydrauliques lors de l'augmentation et de la diminution du débit restitué dans le tronçon court-circuité.

Dans le cas des bassins versant à forte composante nivale, ce sont plutôt les crues de fusion qui remplissent au mieux ces critères (volume, gradients), même si elles sont souvent plus faibles en pointe que les crues automnales.
Les variations de débit et de hauteur en aval du site peuvent être observées par le limnigraphe situé à Cevins. Elles traduisent à la fois une montée rapide des eaux mais également une descente très rapide. Ces deux gradients ont été identifiés comme une source potentielle d'impacts pour la faune [Gayraud et al., 2000]. Les gradients de montée et de descente des débits dans le tronçon court-circuité ont donc été modifiés dans la dernière version de la consigne de chasse pour :

- à la montée, limiter les effets mécaniques liés à la mise en vitesse en permettant aux organismes de regagner les berges ou de s'enfoncer dans le sédiment,

- à la descente, éviter l'échouage des organismes et permettre le tri granulométrique en évitant le dépôt en masse des MES sur toute la section au moment de la baisse des vitesses (Fig. 12).

Les résultats physico-chimiques synthétiques obtenus lors des chasses sont présentés dans le tableau suivant (sur la base de mesures approximativement horaires) (Tableau I).

Les concentrations en oxygène dissous restent largement au-dessus des valeurs critiques pour la faune à l'exception de la première chasse de 1990 où une valeur inférieure à $4 \mathrm{mg} / \mathrm{l}$ a été observée, conduisant à l'arrêt de la chasse et à une seconde chasse sur une crue, un mois plus tard. 
Tableau I. - Quelques valeurs physico-chimiques caractéristiques mesurées lors des chasses sur la retenue d'Aigueblanche.

Table I. - Values of some environmental parameters measured during flushing of the Aigueblanche reservoir.

\begin{tabular}{|c|c|c|c|c|c|c|}
\hline Date & $\begin{array}{c}\text { O2 mini } \\
\mathrm{mg} / \mathrm{l}\end{array}$ & $\begin{array}{c}\text { MES maxi } \\
\mathrm{g} / \mathrm{l}\end{array}$ & $\begin{array}{l}\text { Durée avec } \\
\text { MES }>10 \mathrm{~g} / \mathrm{l}\end{array}$ & $\begin{array}{c}\mathrm{NH}_{4}^{+} \text {maxi } \\
\mathrm{N} \mathrm{mg/l}\end{array}$ & $\begin{array}{c}\text { Q moyen } \\
\mathrm{m}^{3} / \mathrm{s}\end{array}$ & $\begin{array}{c}\text { Flux de MES } \\
\text { évacué } \\
\text { tonnes }\end{array}$ \\
\hline $15 / 06 / 87$ & 8.8 & 23 & 2 h45 & 4.8 & 104 & 100000 \\
\hline $27 / 10 / 87$ & 10.5 & 12 & $1 \mathrm{~h} 00$ & 0.4 & 54 & 45000 \\
\hline $09 / 04 / 90$ & 3.8 & 43 & $10 \mathrm{~h} 00$ & 3.0 & 24 & 24000 \\
\hline $18 / 05 / 90$ & 8.2 & 63 & $20 \mathrm{~h} 00$ & 1.0 & 123 & 300000 \\
\hline $17 / 06 / 91$ & 7.8 & 23 & $8 \mathrm{~h} 00$ & 2.0 & 112 & 68000 \\
\hline $25 / 05 / 94$ & 8.6 & 23 & $13 \mathrm{~h} 00$ & 3.0 & 122 & 210000 \\
\hline $30 / 05 / 95$ & 9.5 & 27 & $11 \mathrm{~h} 00$ & 0.8 & 158 & 120000 \\
\hline${ }^{*} 03 / 11 / 98$ & 6.7 & 42 & $10 \mathrm{~h} 00$ & 3.2 & 100 & 150000 \\
\hline $06 / 05 / 99$ & 9.5 & 12 & $0 \mathrm{~h} 15$ & 0.7 & 95 & 42000 \\
\hline
\end{tabular}

* fait suite à la crue du 26/10/1998 qui a fortement engravé la retenue

Hauteurs limnimétriques aux stations amont et aval d'Aigueblanche durant la chasse de 1995

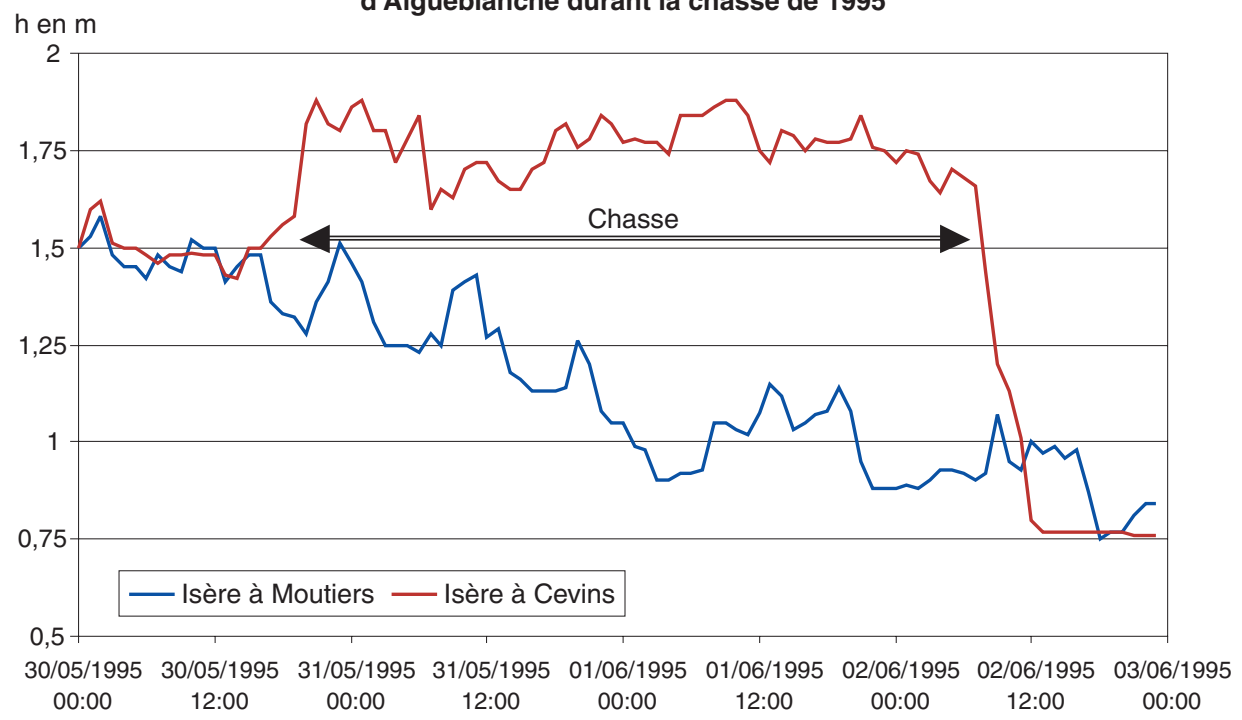

Fig. 12. - Evolution des hauteurs d'eau dans le tronçon court-circuité lors de la chasse de 1995 à Aigueblanche (avant modification de la consigne de chasse).

Fig. 12. - Water level downstream Aigueblanche during the flushing of 1995 (before modifications of operating conditions). 
Ce paramètre a une concentration létale en $96 \mathrm{~h}$ pour $50 \%$ des organismes à $9^{\circ} \mathrm{C}: \mathrm{CL}_{50}-96 \mathrm{~h}\left(9^{\circ} \mathrm{C}\right)$ de l'ordre de $1,7 \mathrm{mg} / \mathrm{l}$ pour la truite [Shepard, 1955 in Magaud, 1997]. Pour des truites fario de 0,2 à $0,9 \mathrm{~g}$, la durée létale pour $50 \%$ des organismes $T_{50}\left(16^{\circ} \mathrm{C}\right)=$ $196 \mathrm{~min}$ pour $\mathrm{O}_{2}=2,6 \mathrm{mg} / \mathrm{l}$ [Garric et al., 1990]

Les valeurs en azote ammoniacal lors des chasses sont plus élevées que les valeurs mensuelles données par le RNDE (Fig. 10). Cependant, les chasses s'accompagnent d'une baisse de $\mathrm{pH}$ qui rend la formation d'ammoniac gazeux dissous très faible $\left(\mathrm{NH}_{3}\right.$ maxi $\left.=0,02 \mathrm{mg} / \mathrm{l}\right)$, contrairement aux mesures en étiage de Février $\left(\mathrm{NH}_{3} \operatorname{maxi}=0,1 \mathrm{mg} / \mathrm{l}\right)$. Ce paramètre a une $\mathrm{CL}_{50}(96 \mathrm{~h})$ de l'ordre de 0,3 à $0,5 \mathrm{mg} / \mathrm{l}$ pour des truites arcen-ciel de $10 \mathrm{~g}$ [Thurston, 1981 in Magaud, 1997], mais des effets sur les communautés aquatiques sont signalés pour des œufs de truites [Niederlehner \& Cairns, 1990].

Seules les MES présentent des teneurs importantes, parfois proches des limites de toxicité à court terme pour la faune. Les estimations de $\mathrm{CL}_{50}$ pour les MES (de $10 \mathrm{~h}$ à $20 \mathrm{~h}$ selon les granulométries) sont de l'ordre de $30 \mathrm{~g} / \mathrm{l}$ [Cardinal, 1988], des teneurs à $17 \mathrm{~g} / \mathrm{l}$ pendant plus de 3 heures conduisent à $10 \%$ de mortalité sur des truitelles [Garric et al., 1990].

$\mathrm{Si}$ on tient compte des synergies entre la toxicité des MES et de l'oxygène dissous [Garric et al., 1990], pour la majorité des chasses, on se situe à un niveau de toxicité qui atteint ou dépasse légèrement l'iso- chrone $(2 \mathrm{~h})$ de mortalité de $10 \%$ de truitelles de $6 \mathrm{~cm}$. Les deux chasses de 1990 et celle de 1998, (concentrations en MES au-delà de $40 \mathrm{~g} / \mathrm{l})$, sont en théorie beaucoup plus pénalisantes en dépassant probablement la $\mathrm{CL}_{50}$ sur la deuxième chasse de 1990 . Mais ces deux opérations font suite à plusieurs années sans chasse possible compte tenu des contraintes sur les débits de chasse.

L'effet toxique induit par ce paramètre est cependant exagéré par surestimation des concentrations car les prélèvements sont effectués dans la veine d'eau principale (pour être représentatifs des flux) et non dans les zones latérales de refuge où la concentration est plus faible [ENSAT, 1988].

\section{5 ÉVOLUTION DES PARAMÈTRES BIOLOGIQUES}

\subsection{Evolution des indices biologiques}

Les IBGN (Gay, 1992) sont mesurés (Fig. 1) de manière régulière sur les stations du RNDE (Centrons et Feissons) et de manière plus circonstanciée lors des chasses (Amont Isère et Doron, Aigueblanche, Morel, la Léchère): une mesure est effectuée systématiquement en début de printemps avant la période favorable aux chasses et si une chasse a eu lieu, une mesure est effectuée a posteriori, lorsque les conditions hydrauliques le permettent (en général après la période de fusion nivale pour les chasses printanières). 


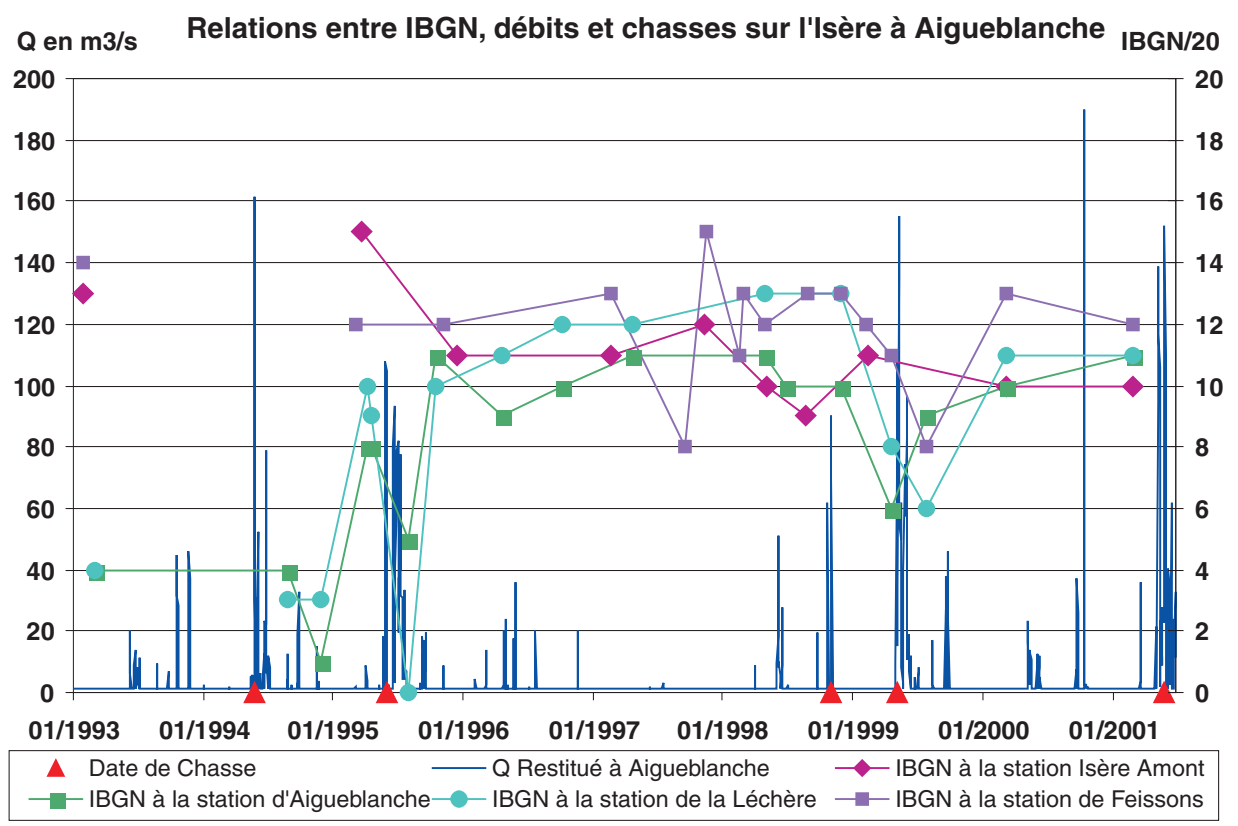

Fig. 13. - Relations entre IBGN, débits et chasses sur l'Isère à Aigueblanche.

Fig. 13. - Relationships between IBGN, flowrates and flushings on Isère river in Aigueblanche station.

Après une première période très perturbée jusqu'en 1995, du Pont d'Aigueblanche jusqu'à la Léchère, les IBGN reviennent à des niveaux similaires à l'amont ou à l'aval lointain (Fig. 13). En 1999, les valeurs chutent à nouveau mais dans une moindre mesure avant de se restabiliser dans la gamme 10-12. Ces baisses d'indice semblent liées aux épisodes de crues et de chasse sans qu'on puisse préciser la part respective de chacun des phénomènes. Par contre, après la période 1996-1998, très calme sur le plan hydrologique et sans chasse, les IBGN ne remontent pas au-delà de 12 à 13 suggérant qu'un autre impact devient alors prépondérant.
Une mesure complémentaire (Morel et Aigueblanche) sans chasse mais après deux périodes de déversement (à 51 puis $28 \mathrm{~m}^{3} / \mathrm{s}$ ) a permis de mettre en évidence le rôle de ces épisodes malgré leur faible amplitude. La perte de la diversité faunistique est très sensible. Elle se traduit par une baisse de l'IBGN de 5 points à la station du Morel car les Leuctridae qui définissent le groupe indicateur disparaissent (Tableau II). L'impact hydraulique qui résulte de la mise en vitesse par des débits peu habituels d'un tronçon fortement endigué semble donc devoir être privilégié sur l'impact physico-chimique des chasses. 
Tableau II. - Evolution de I'IBGN aux stations du Morel et d'Aigueblanche avant et après une période de déversement dans le tronçon court-circuité.

Table II. - Time serie of Invertebrate Index at the Morel and Aigueblanche stations before and after an overflow discharge downstream the Aigueblanche reservoir.

\begin{tabular}{|c|c|c|c|c|c|c|}
\hline & \multicolumn{3}{|c|}{ Station du Morel } & \multicolumn{3}{c|}{ Station d'Aigueblanche } \\
\hline Date & IBGN & $\begin{array}{c}\text { Groupe } \\
\text { Indicateur }\end{array}$ & $\begin{array}{c}\text { Diversité } \\
\text { faunistique }\end{array}$ & IBGN & $\begin{array}{c}\text { Groupe } \\
\text { Indicateur }\end{array}$ & $\begin{array}{c}\text { Diversité } \\
\text { faunistique }\end{array}$ \\
\hline Avant : 28/4/98 & $\mathbf{1 3}$ & 7 & 22 & $\mathbf{1 1}$ & 7 & 16 \\
\hline Après : 30/6/98 & $\mathbf{8}$ & 4 & 14 & $\mathbf{1 0}$ & 7 & 12 \\
\hline
\end{tabular}

\section{2 Évolution des populations piscicoles}

L'évolution des peuplements piscicoles est observable à partir des pêches réalisées annuellement dans le cadre des suivis de chasse sur 3 points répartis dans le tronçon courtcircuité. Sur les 2 stations amont (Aigueblanche et la Léchère), l'Isère est fortement endiguée mais facile à échantillonner au débit réservé. Sur la station aval (Esserts-Blays), elle présente encore différents bras ce qui est plus conforme au style fluvial initial, mais elle devient très délicate à échantillonner dès que les torrents du bassin versant en aval d'Aigueblanche soutiennent les débits.

Les espèces dominantes sont la truite fario soumise à un alevinage important, la truite arc-en-ciel présente à cause de déversements réguliers et quelques populations relictuelles des espèces d'accompagnement (vairon, chabot, blageon, loche) dont certaines sont sans doute en limite amont de leur aire d'extension initiale au niveau d'Aigueblanche.
Les mesures faites dans le cadre des suivis de chasses montrent, pour la truite fario, des niveaux en biomasse faibles à très faibles, sauf en 1998 (Fig. 14). Cette année spécifique semble la résultante d'une progression constante depuis 1996. Si les résultats ne sont pas tout à fait concordants, ils sont néanmoins fortement covariants sur toutes les stations, laissant penser à une dynamique globale à l'échelle du tronçon. La station d'Aigueblanche apparaît cependant plus faible que les autres points même en 1998, ce qui peut s'expliquer soit par la proximité du barrage soit par un milieu beaucoup moins favorable (pauvreté en caches).

A cette variabilité en biomasse correspond également une variabilité dans la structure des populations. Les figures $15 \mathrm{a}$ et $15 \mathrm{~b}$ donnent les histogrammes de taille des populations de truites à la station d'Esserts Blays, en Avril et en Novembre 1998 (des résultats similaires sont observés sur les autres stations). Si la structure d'Avril 1998 présente une population classique de juvéniles et quelques adultes encore présents, il n'en va pas de même de la structure 


\section{Evolution spatiale et temporelle de la biomasse de truite fario}

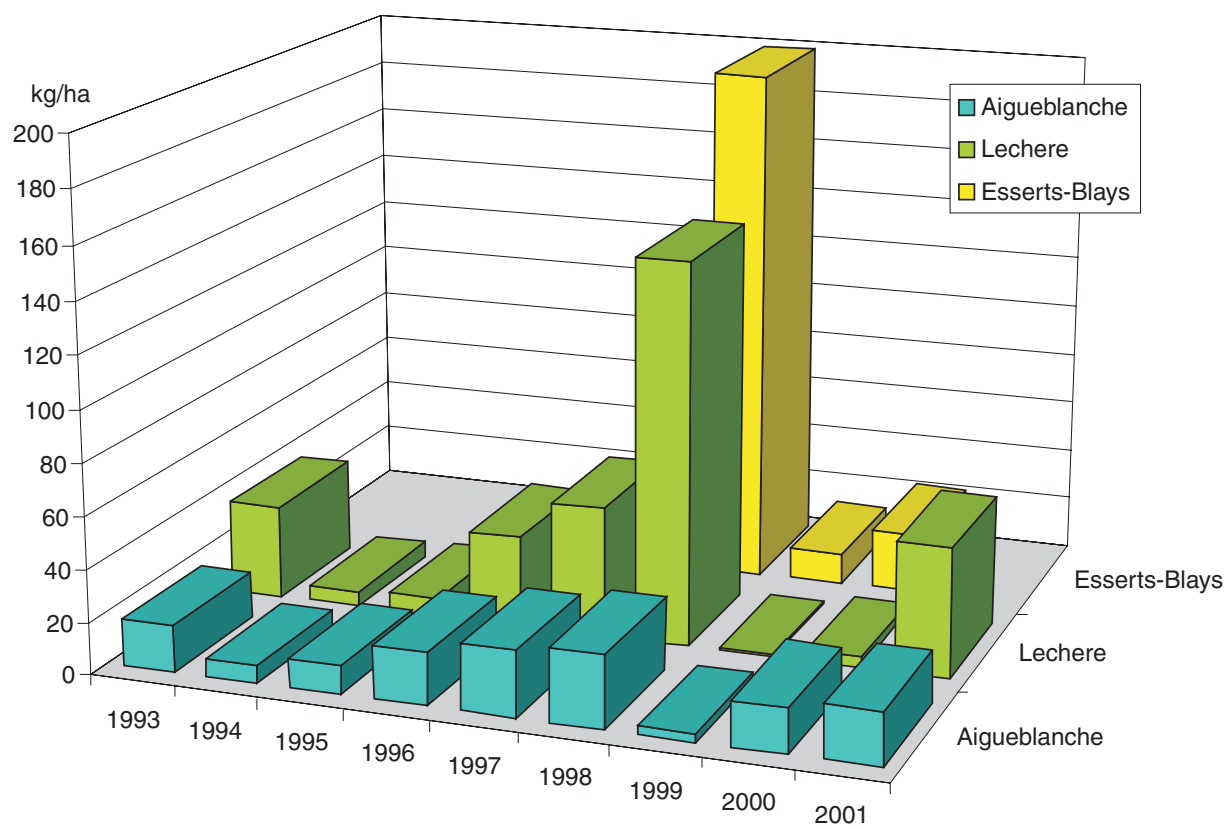

Fig. 14 - Evolution des biomasses de truites fario en aval d'Aigueblanche.

Fig. 14 - Biomass of brown trout downstream Aigueblanche reservoir.

de Novembre 1998 avec une population quasi relictuelle sauf pour les alevins. Entre ces deux pêches, il y a les épisodes de crues de 1998, associés à une chasse en Novembre, la saison de pêche 1998, voire d'autres impacts non connus.

On constate, de manière générale, sur tous les points de mesure que ce sont les adultes qui semblent résister le moins bien aux épisodes de crues et chasses en 1998, alors qu'ils sont les plus à même de résister aux fortes vitesses. Cela peut s'interpréter soit par des mouvements migratoires à l'échelle du bassin versant entre les deux périodes, soit par une pression de pêche très élevée dans le tronçon à débit réservé. Dans le bassin versant de l'Isère, ces tronçons sont souvent parmi les rares secteurs qui présentent des eaux moins torrentueuses et moins turbides, notamment à la fonte des neiges, et qui, de ce fait, sont plus favorables à I'halieutisme.

\subsection{Interprétation des données biologiques}

Dans un tel contexte écologique, les données biologiques deviennent 
Histogramme des classes de tailles à Esserts Blays en Avril 1998

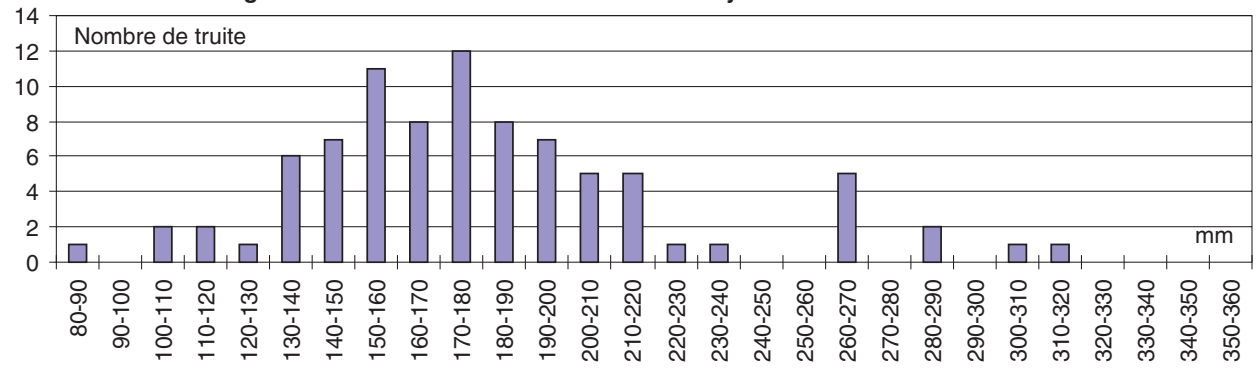

Histogramme des classes de tailles à Esserts Blays en Novembre 1998

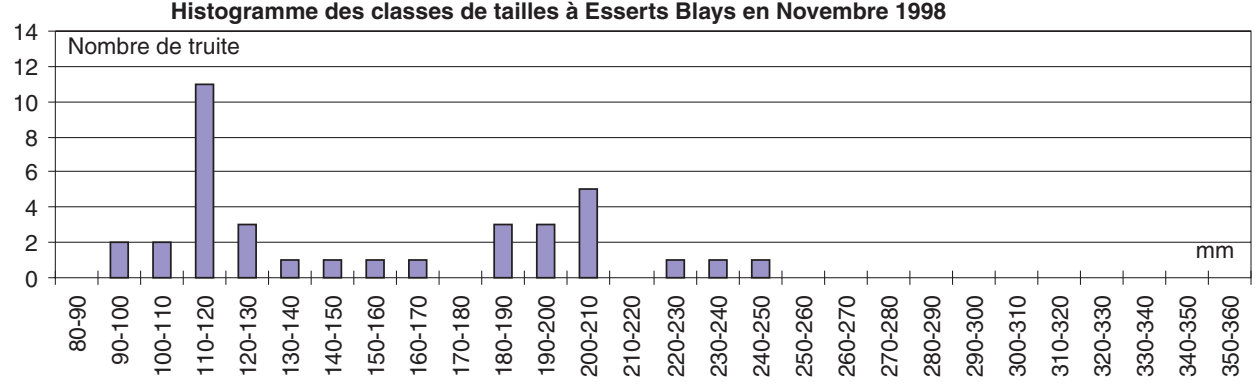

Fig. 15a et 15b. - Evolution des classes de tailles pour la truite fario à la station d'Esserts Blays. Fig. 15a et 15b. - Class size distribution for brown trout at Esserts Blays station.

très délicates à interpréter particulièrement lorsque les mesures sont focalisées sur un seul impact. Au fil des rapports environnementaux des suivis de chasse, les interprétations sont apparues de plus en plus difficiles à étayer, notamment parce qu'il était admis que, comme pour les vidanges, les impacts étaient essentiellement liés aux caractéristiques physicochimiques des eaux restituées en aval de la retenue [Cardinal, 1988], [Poirel et al., 1994].

Dans le contexte spécifique d'Aigueblanche, l'interprétation par comparaison avec un écosystème amont est également très délicate car les milieux sont très différents en amont et en aval de la retenue. De plus, l'amont subit également nombre d'impacts autres que les chasses.

Ces difficultés d'interprétation ont rendu nécessaire, a minima, le recensement de toutes les causes possibles d'impact et leur analyse croisée. Celles-ci sont répertoriées dans le Tableau III.

De par la variabilité des observations biologiques, il est probable que des événements ponctuels, mais significatifs, viennent fortement perturber l'écosystème de manière récurrente venant s'ajouter à des facteurs à plus long terme qui conditionnent les meilleurs états possibles à une date donnée. 
Tableau III. - Nature des différents impacts sur l'Isère en aval d'Aigueblanche, évolution temporelle de chaque impact et liaisons synergiques ou antagonistes avec les autres impacts.

Table III. - Nature of environmental impacts on Isère downstream Aigueblanche reservoir, temporal evolution of each impact and interactions with other impacts.

\begin{tabular}{|c|c|c|}
\hline Nature des impacts & Evolution temporelle & Liaison avec les autres impacts \\
\hline $\begin{array}{l}\text { Evolution morphodynamique } \\
\text { et endiguement }\end{array}$ & $\begin{array}{l}\text { Dégradation par ajouts } \\
\text { réguliers de points durs et } \\
\text { augmentation des capacités } \\
\text { routières (tourisme) }\end{array}$ & $\begin{array}{l}\text { Habitats piscicoles réduits en } \\
\text { crue par mise en vitesse et } \\
\text { entraînement des organismes. }\end{array}$ \\
\hline $\begin{array}{l}\text { Evolution des températures } \\
\text { de l'air donc probablement } \\
\text { des températures de l'eau }\end{array}$ & $\begin{array}{l}\text { Hausse notable la dernière } \\
\text { décennie des températures } \\
\text { de l'air printanières }\end{array}$ & $\begin{array}{l}\text { Crues de printemps plus tôt } \\
\text { dans la saison (impact sur } \\
\text { l'émergence des alevins) et } \\
\text { peut être perturbations de } \\
\text { certains cycles biologiques? }\end{array}$ \\
\hline $\begin{array}{l}\text { Prélèvements de granulats } \\
\text { en rivière }\end{array}$ & $\begin{array}{l}\text { Probablement en diminution } \\
\text { mais le maintien des lignes } \\
\text { d'eau est souvent prioritaire } \\
\text { sur la liberté de divagation } \\
\text { de la rivière. }\end{array}$ & $\begin{array}{l}\text { Favorise l'incision du lit avec } \\
\text { l'endiguement et rend les } \\
\text { affluents peu accessibles } \\
\text { (confluences perchées). }\end{array}$ \\
\hline Rejets de métaux lourds & $\begin{array}{l}\text { Amélioration observée ces } \\
\text { dernières années }\end{array}$ & \\
\hline $\begin{array}{l}\text { Rejets domestiques : } \\
\mathrm{NH}_{4}^{+}-\mathrm{NH}_{3} \text { dans un fond } \\
\text { géochimique de } \mathrm{pH} \text { élevé }\end{array}$ & $\begin{array}{l}\text { Dégradation observée avec } \\
\text { le développement du } \\
\text { tourisme - peu de mesures } \\
\text { lors des périodes à risques }\end{array}$ & $\begin{array}{l}\text { Dilution plus faible en débit } \\
\text { réservé, dilution plus élevée } \\
\text { lors des turbinages de la Bathie }\end{array}$ \\
\hline $\begin{array}{l}\text { MES lors des crues et des } \\
\text { chasses }\end{array}$ & $\begin{array}{l}\text { Amélioration en chasse } \\
\text { mais fonction des dépôts } \\
\text { dans la retenue eux mêmes } \\
\text { fonction des apports et de } \\
\text { la fréquence des chasses }\end{array}$ & $\begin{array}{l}\text { Impacté par des laves } \\
\text { torrentielles conséquentes sur } \\
\text { les affluents amont ou aval. }\end{array}$ \\
\hline $\begin{array}{l}\text { Hydraulique en crue et en } \\
\text { chasse }\end{array}$ & $\begin{array}{l}\text { Amélioration des gradients } \\
\text { lors des chasses }\end{array}$ & $\begin{array}{l}\text { Synergie forte avec } \\
\text { l'endiguement (vitesse très } \\
\text { élevées) et les difficultés } \\
\text { d'accès aux affluents (zones de } \\
\text { refuges inaccessibles) }\end{array}$ \\
\hline $\begin{array}{l}\text { Halieutisme (prélèvement } \\
\text { des adultes, introduction de } \\
\text { truites farios ou arc-en-ciel). }\end{array}$ & $?$ & $\begin{array}{l}\text { Pression de pêche très élevée } \\
\text { dans le tronçon court circuité, } \\
\text { surtout les années de forte } \\
\text { fusion nivale (eaux plus claires, } \\
\text { plus attractives pour la pêche) }\end{array}$ \\
\hline
\end{tabular}

L'hydrologie et l'hydraulique semblent jouer un rôle prépondérant d'autant que l'endiguement ne permet plus de passer de forts débits dans des conditions de vitesse acceptable pour la faune. En paral- lèle, les refuges sont moins nombreux, tant au niveau des affluents rendus inaccessibles par l'incision du lit (confluences perchées) que par la nature même des berges endiguées. 
La période de fin 1995 à mi 1998, de faible hydraulicité, a été la plus favorable aux populations piscicoles et aux invertébrés qui ont atteint simultanément les plus fortes valeurs. A contrario, les périodes de très forte hydraulicité sont celles qui conduisent à des valeurs biologiques très faibles. Comme elles sont à la fois les périodes les plus favorables au transit solide et l'occasion de réaliser des chasses, les deux impacts sont difficilement dissociables. La physicochimie lors des chasses, contrairement aux idées initiales, ne paraît pas être un impact direct majeur. II subsiste néanmoins trois impacts propres aux chasses:

- les gradients hydrauliques de montée des débits de chasse favorisent l'entraînement des organismes - cela a été corrigé par les nouvelles consignes administratives qui tendent à retrouver maintenant les valeurs « naturelles » au moins en début de chasse,

- les gradients de fin de chasse contribuent à figer le lit de l'Isère en arrêtant brusquement le transport solide par retour au débit réservé. Eux aussi ont été adaptés mais la remise en fonctionnement de l'aménagement hydraulique de Randens limite les possibilités techniques au voisinage du débit réservé (débit minimum à respecter sur chaque groupe),

- le report temporel des grands événements de transport solide intervenus en amont jusqu'à la chasse suivante : ainsi, la crue de l'Arbonne de Juillet 1996 a causé des modifi- cations notables dans le lit de l'Isère en aval de Bourg-SaintMaurice, mais l'Isère en aval d'Aigueblanche n'a pas été touchée entre 1996 et 1998 ; par contre, la chasse de Novembre 1998 s'est traduite par des dépôts conséquents de granulométrie assez inhabituelle (sables à graviers) uniformisant les habitats et colmatant les rares caches de bordure au niveau des enrochements [SA Gestion Environnement, 1998].

Néanmoins, même dans les conditions hydrauliques les plus favorables pour la faune, les IBGN sont encore à des niveaux moyens. Ils se sont cependant nettement améliorés depuis 1993 et on peut supposer, mais sans certitude, que la réduction des rejets métalliques a eu un impact positif sur la faune benthique.

Quant aux autres impacts résiduels, les facteurs explicatifs ne sont pas clairement identifiables avec les données actuelles. Les valeurs d'ammoniac en saison touristique seraient, par exemple, à contrôler à des pas de temps plus fins qu'une valeur mensuelle compte tenu des niveaux atteints et de la sensibilité des œufs de truite à ce polluant durant cette période.

\section{DISCUSSION ET CONCLUSIONS}

Dans le cadre du retour d'expérience sur les chasses hydrauliques pratiquées sur la retenue d'Aigueblanche, la limite dans la compréhension des processus biologiques est 
liée au manque de cohérence globale des informations disponibles : certaines variables sont acquises dans le cadre de programmes génériques de surveillance de l'état du milieu tel le RNDE (beaucoup de paramètres mais représentation spatiale et temporelle faible), alors que d'autres sont acquises dans le cadre de suivi d'impacts spécifiques tel le suivi des chasses (moins de paramètres mais mesures effectuées dans des situations spécifiques à impact potentiel avec une meilleure représentation spatiale et temporelle). Certaines informations sont également totalement absentes ou peu accessibles pour un acteur particulier.

II devient alors tentant d'interpréter toute variation biologique comme une conséquence de l'impact étudié en faisant abstraction des synergies ou des antagonismes entre les différentes sources d'impacts.

Quelques processus faisant intervenir des effets du type "rétroaction " vont parfois à l'encontre des raisonnements habituels: compte tenu de l'endiguement actuel de l'Isère, rivière à très haute énergie, c'est paradoxalement les années où le tronçon court-circuité reste en débit réservé que les populations piscicoles atteignent leurs plus fortes valeurs. En situation "naturelle », avec un débit égal au module, les habitats deviendraient singulièrement réduits de par la forme des sections puisque sur $4 / 5^{\text {ème }}$ de la largeur, la vitesse moyenne dépasse déjà $1 \mathrm{~m} / \mathrm{s}$. II y a donc un antagonisme entre l'im- pact « débit réservé », favorable lorsqu'il n'y a pas de déversements et l'impact « endiguement ». Par contre, à fort débit, ils interviennent en synergie: d'une part la crue a des conséquences plus fortes sur un système en débit réservé, d'autre part, l'endiguement augmente les vitesses tout en réduisant les possibilités de refuge latéral ou les accès aux affluents.

Dans ce type d'écosystème, seule une étude concertée entre tous les acteurs, conduite par itérations successives sur plusieurs années, et sans a priori sur les impacts structurants permettrait de définir le ou les facteurs qui conditionnent les peuplements et d'en atténuer les effets. Elle devrait définir également une variabilité "naturelle» (donc acceptable?) de ces écosystèmes montagnards soumis à des événements particuliers souvent exacerbés par rapport à la majorité des systèmes classiquement étudiés (crues, laves torrentielles, tourisme...). Seules ces connaissances pourraient permettre de sortir d'une logique de "réaction à chaud » où les questions environnementales sont posées indépendamment à chaque gestionnaire, traitées par successions de contraintes particulières, sans cohérence ni contrôle d'efficacité. Enfin, elles pourraient faire prendre conscience que «bon état écologique " n'est pas synonyme d'optimum biologique et que la variabilité spatiale et temporelle des peuplements est une réponse normale aux facteurs écologiques. 


\section{RÉFÉRENCES BIBLIOGRAPHIQUES}

Anville (J.B. d'), 1697-1782 - Carta generale de Stati di Sua Altezza reale [Etats de savoie] ; Cote BNF : Ge DD 2987 Reprod. Sc 96/833-844.

Bovio (H.) \& Poirel (A.), 1998 - Retour d'expérience sur les vidanges et chasses hydrauliques effectuées sur la retenue d'Aigueblanche sur I'Isère Document EDF, UP Alpes.

Cardinal (H.), 1988 - Procédure LIVRE : Bilan des recherches et recommandations en matière de vidanges de retenues - Document EDF HE 31/88-20.

ENSAT - Laboratoire d'Ichtyologie Appliquée, 1988 : Etude de la toxicité des sédiments de la retenue de Plan d'Arem sur la population de truites Document ENSAT pour EDF - GRPH Pyrénées.

Garric (J.), Migeon (B.) \& Vindimian (E.), 1990 - Lethal effects of draining on brown trout. A predictive model based on field and laboratory studies. Water Research Vol. 24 no1 pp. 59-65.

Gay (C.), 1992 - Indice Biologique Global Normalisé IBGN - NF T 90-350 Guide Technique Cabinet Gay Environnement \& Agence de l'Eau.

Gayraud (S.), Herouin (E.), Philippe (M.), Jacob (F.), Souchon (Y.) \& Valentin (S.), 2000 : Impacts de la vidange de la retenue de Choranche (Bourne) sur la faune aquatique. Rapport final - Document Cemagref Lyon.

IGN, 1998 - Carte 3532 OT au 25 000ѐme.

Koulinski (V.), 1999 - La crue de l'Arbonne à Bourg-Saint-Maurice (73) - Site Internet ERTM : http : //vkoulinski.chez.tiscali.fr/crues.htm.

Magaud (H.), 1997 - Modélisation des effets létaux de rejets pluviaux urbains sur la truite Arc en Ciel à l'aide d'essais de laboratoire - Thèse Université Cl. Bernard - Lyon 1 - 19/9/1997.
Ministère de l'Equipement et Voies Navigables de France - Etude 2001 - Evaluation écotoxicologique de sédiments contaminés ou de matériaux de dragage - Synthèse du rapport d'étude (Avril 2001).

Niederlehner (B.R.) \& Cairns (J.), 1990 Effect of Ammonia on periphytic communities - Env. Pollution n66 (1990) pp. 201-221.

Paquet (E.) \& Garçon (R.), $2001-$ Caprice du climat et de l'hydrologie en Haute Durance - Colloque SHF « Variations climatiques et Hydrologie» Paris - Décembre 2001.

Poirel (A.), Vindimian (E.) \& Garric (J.), 1994 - Gestion des vidanges de réservoirs. Mesures prises pour préserver l'environnement et retour d'expérience sur une soixantaine de vidanges. CIGB 18éme Congrès des grands barrages. Durban, 1994 pp. 321-349.

Poirel (A.) \& Bessy (P.), 2000 - Etude du transport solide dans le bassin de la Coche (74) - Document EDF-DTG.

Poirel (A), Carrel (G.) \& Olivier (J.M.), 2001 - Illustration de la complémentarité des chroniques environnementales dans l'étude d'un hydrosystème fluvial - Régime thermique et peuplements piscicoles du Rhône. Actes du Colloque Lyon Fleuves, 2001.

SA Gestion de l'Environnement: Rapports de suivi des chasses de 1994, 1995, 1998, 1999 - Documents EDF Energie Alpes.

Service des Grandes Forces Hydrauliques (1900-1913) : Annuaires hydrologiques, cartographiques et de nivellements - Documents Ministère de l'Agriculture - Direction de l'Hydraulique Tome IV.

Smith (S.L.), MacDonald (D.D.), Keenleyside (K.A.) \& Gaudet (C.L.), 1996 The development and implementation of Canadian Sediment Quality Guidelines - pp. 233-249 in Development and Progress in sediments Quality Asses- 
sement - Edited by M. Munawar \& G. Wasson (J.G.), Malavoi (J.R.), Maridet Dave.

Tourasse (P.), 1989 - Projet de Roselend - Cevins - Evaluation des crues extrêmes de l'lsère à Aigueblanche $\left(1582 \mathrm{~km}^{2}\right)$ et Cevins (1780 km²). Do(L.), Souchon (Y.) \& Paulin (L.), 1995 Impacts écologiques de la chenalisation des rivières - CEMAGREF EPTEAU Min Environnement Commande DE 30/93. cument EDF DTG. 
